\title{
Relaciones asociativas entre las creencias acerca del uso social de las drogas y el consumo en estudiantes jóvenes
}

\author{
Associative Relationships between Beliefs about the Social Use \\ of Drugs and the Consumption among Young Students
}

Recibido: febrero 21 de 2012 | Revisado: octubre 20 de 2012 | Aceptado: diciembre 18 de 2012

\author{
Humberto M. Trujillo* \\ Universidad de Granada, España \\ José M. MARTíneZ-GonZÁLEZ \\ Centro Provincial de Drogodependencias, Granada, España \\ CRISTINA VARGAS \\ Universidad de Granada, España
}

doi:10.11144/Javeriana.UPSY12-3.race

Para citar este artículo: Trujillo, H. M., MartínezGonzález, J. M \& Vargas, C. \& , . (2013). Relaciones asociativas entre las creencias acerca del uso social de las drogas y el consumo en estudiantes jóvenes. Universitas Psychologica, 12(3), 875-885. doi:10.11144/Javeriana.UPSY12-3.race

* Universidad de Granada, Facultad de Psicología. Campus Universitaro de Cartuja s. n. 18071, Granada, España. Teléfono: +34958 246271. E-mail: humberto@ugr.es

\section{RES UMEN}

El objetivo de este estudio fue conocer el contenido de las representaciones sociales acerca del consumo de drogas en adolescentes y adultos jóvenes, y si tales representaciones favorecían y, por lo tanto, predecían el consumo de las mismas. Para esto, se seleccionaron seis creencias acerca del uso social de las drogas -tales como tomar alguna droga es normal-, considerándolas como indicadores de dichas representaciones sociales. La muestra estuvo compuesta por 417 alumnos del Ciclo Formativo de Grado Medio y Programa de Garantía Social pertenecientes a distintos centros educativos de la ciudad de Granada, considerados de alto riesgo para el consumo. Los resultados mostraron que las creencias evaluadas estaban presentes entre los participantes, que predecían el consumo de drogas legales e ilegales y que estaban asociadas sobre todo al consumo de cannabis. En conclusión, en los programas preventivos sería de gran utilidad tener en cuenta las representaciones sociales sobre el uso de drogas.

Palabras clave autores

Consumo de drogas, creencias, jóvenes, representaciones sociales.

Palabras clave descriptores

Psicología de la salud, sustancias psicoactivas, adicción.

\section{A B S T R A C T}

The objective of the study was to know the content of social representations about the consumption of drugs and also if those representations contributed and predicted the consumption of drugs among young people. Six beliefs about the social use of drugs were selected, such as whether it is common to take drugs. These beliefs were considered indicators of those social representations. The study was carried out among 417 students of Associate Degree and Social Guarantee Program from different Educational Centres of Granada, who were considered high risk drug users. Results showed that evaluated beliefs were present among young people, these beliefs predicted the consumption of legal and illegal drugs and were specially associated with the consumption of cannabis. Thus, social representations about the consumption of drugs should be considered for prevention programs.

Key words authors

Beliefs, Consumption of Drugs, Social Representations, Young people.

Key words plus

Health Psychology, Psychoactive Drugs, Addiction. 
La teoría de las representaciones sociales se muestra como una referencia útil y obligada para el estudio de las drogodependencias (Megías, 1999), ya que desde ella se predice que las personas consumen drogas, entre otras cosas, porque desarrollan ciertas creencias acerca de la extensión de su uso y de los efectos que producen, influenciadas por su ambiente social.

El concepto de 'representación social' fue acuñado con el fin de denotar el fenómeno social como una realidad que guarda relación con el comportamiento de las personas que conforman los grupos sociales, siendo Moscovici (1986) el referente fundamental en este campo de estudio. Megías, Comas, Elzo, Navarro y Romaní (2001), partiendo de aportaciones como la de Moscovici, consideran las representaciones sociales como un conjunto ordenado de valores y creencias que permiten a las personas comunicarse y, así, orientarse en el contexto social al que pertenecen, comprender sus acciones y explicar y defender su identidad. Además, para acotar resumidamente el concepto de representaciones sociales se señalan las tres características principales que las definen de acuerdo con Wagner y Elejabarrieta (1995): (1) se construyen socialmente; (2) muestran una amplia homogeneidad y distribución en el seno de los grupos implicados; y (3) como conocimiento de naturaleza social suponen una alta relación entre procesos y contenidos de pensamiento.

De esta forma, las representaciones sociales favorecen el desarrollo de una fuerte asociación entre el contenido de las mismas y el comportamiento de las personas que las comparten, generando un tipo específico de conocimiento cuyo papel es crucial en la forma en que las personas piensan y organizan su vida. Tanto es así que la cognición social debe ser especialmente atendida en el estudio de las variables que determinan la salud y el bienestar social, ya que mediante el proceso de influencia social es posible establecer y cambiar las actitudes, valores y comportamientos de las personas (Becoña, 2002), lo que podría ser muy importante en la prevención de las drogodependencias. En esta misma línea, cabe hacer explícito que en un estudio de Moral, Sirvent, Ovejero y Rodríguez (2004) se confirma la eficacia de estrategias preventivas tendentes al fortalecimiento de actitudes no permisivas para promover cambios hacía el no consumo de drogas.

En otras palabras, las representaciones sociales, además de estar constituidas por valores, nociones y creencias, también implican actitudes que a su vez se relacionan con la conducta (Megías et al., 2001). Sin embargo, en el análisis de la relación entre representaciones sociales y actitud, Myers (2000) muestra cómo desde la psicología social no se ha podido constatar la relación inequívoca entre actitud y conducta. No obstante, y a pesar de lo anterior, informa que las actitudes pueden predecir la conducta si: (1) se minimizan otras influencias; (2) la actitud es específica respecto a la acción; y (3) la actitud es fuerte, porque es recordada por algo o porque se ha formado de forma intensa. Por este motivo, es necesario el estudio de las variables que hacen más probable el inicio en el consumo de drogas, y evaluar y conocer las actitudes hacia el consumo de las mismas, dado que las actitudes representan tendencias o predisposiciones de actuación que, por otro lado, están asociadas a determinadas expectativas sobre el comportamiento de los demás (Observatorio Europeo de las Drogas y las Toxicomanías, 2003; Páez, Basabe, Igartua, Iraurgi $\&$ Valdoseda, 1992b).

Así mismo, Basabe y Páez (1992) otorgan una gran importancia a las representaciones sociales para la comprensión del consumo de alcohol entre los jóvenes, como fenómeno claramente social. Por otro lado, en un trabajo de Pons, Berjano y García (1999) se constató la relación entre consumo de alcohol y actitudes manifiestas hacia ese consumo. También se ha encontrado que la creencia acerca de la cantidad de alcohol que consumen otros estudiantes está asociada con un mayor consumo de dicha droga (Day-Cameron, Muse, Hauenstein, Simmons \& Correia, 2009). Además, parece que las actitudes de las personas acerca de las drogas están relacionadas con el consumo de las mismas (McCuller, Sussman, Dent \& Teran, 2001), que tanto las actitudes de los adolescentes acerca del consumo de drogas como las normas de los grupos de iguales cambian antes de que estos comiencen a consumir (Jessor \& Jessor, 1977) y que existe un 
cambio general de actitudes, condicionado por las creencias, previo al cambio de conducta (Pisano \& Rooney, 1988).

Se ha demostrado que el consumo de éxtasis está modulado en gran medida por el tipo de representación social que tienen las personas acerca de los efectos de esta droga (Calafat et al., 1998; Calafat et al., 2003), ya que el consumo de la misma está asociado con actitudes favorables hacia éste (Martins, Storr, Alexandre \& Chilcoat, 2008; Wu, Liu \& Fan, 2010). En un estudio llevado a cabo en varios países europeos, se pudo observar que conociendo las expectativas de las personas sobre los efectos del éxtasis o de los peligros percibidos de su consumo se podía determinar el tipo de conducta que mostrarían. Así, conocer esta actitud o, lo que es lo mismo, conocer el contenido de las representaciones sociales genera cierta capacidad predictiva de la conducta de consumo. Al menos así lo han comprobado Calafat et al. (1998). Por lo tanto, tales representaciones sociales parecen tener una influencia directa en las personas, debiéndose tener en cuenta que el contenido, la estructura y la relación entre estas y la conducta está también determinada por el momento histórico y contexto social en el que se encuentran dichas personas (Megías et al., 2001; Ramos, Moreno, Rivera \& López, 2011; Szmigin, Bengry-Howell, Griffin, Hackley \& Mistral, 2011; Vargas \& Trujillo, 2006), además del contexto ambiental personal (Trujillo, OviedoJoekes \& Vargas, 2005, 2006).

Así las cosas, los objetivos de esta investigación fueron los siguientes: (1) evaluar si ciertas creencias acerca del consumo de drogas (p. ej., toda la gente consume drogas; tomar alguna droga es normal; si controlas no tiene porque pasarte nada; deberían legalizar todas las drogas; deberían legalizar algunas drogas; tenemos que aprender a convivir con las drogas) pueden ser consideradas como indicadores de las representaciones sociales acerca del uso comunitario de las drogas; (2) estudiar si las creencias acerca del uso social de las drogas predecía su consumo; (3) estudiar la percepción de incidencia del consumo de drogas que tenían los participantes, tanto en el instituto al que pertenecían como en la ciudad en la que vivían, y si esta percepción estaba relacionada con sus creencias acerca del consumo social; y (4) estudiar si el hecho de tener amigos consumidores de drogas predecía el grado con el que los participantes se identificaban con las creencias acerca de su uso social.

\section{Método}

\section{Participantes}

La muestra estuvo compuesta por 417 alumnos que cursaban sus estudios en algún Ciclo Formativo de Grado Medio o Programa de Garantía Social en tres institutos de la ciudad de Granada, considerados de alto riesgo para el consumo debido a su perfil de fracaso académico. El $60.1 \%$ era de sexo masculino $(N=251)$ y el $39.9 \%$, de sexo femenino $(N=166)$. La edad media fue de 17.88, siendo el rango de 15 a 25 años. El procedimiento de muestreo fue de tipo aleatorio por conglomerados.

\section{Instrumento de medida}

En el presente estudio se administró el cuestionario elaborado por Martínez-González, Trujillo y RoblesLozano (2007) compuesto por tres apartados. En el primero, se obtenía información sobre variables sociodemográficas (edad y género), sobre la frecuencia de consumo de drogas legales e ilegales en los participantes, así como sobre el número de amigos consumidores (respuesta tipo Likert, donde 1 significaba: ninguno, 2: muy pocos, 3: algunos, 4: muchos y 5: todos). En un segundo apartado, se preguntaba a los participantes por la percepción que tenían acerca de la incidencia del consumo de drogas legales (tabaco y alcohol) e ilegales ('porros', drogas de diseño, cocaína, heroína y otros opiáceos) tanto en sus institutos como en su ciudad. Estos tenían que indicar la cantidad de personas que, según ellos, consumían habitualmente drogas en una escala de tipo Likert, cuyos valores de respuesta eran: $1=$ ninguno, $2=$ muy pocos, $3=$ algunos, $4=$ bastantes y $5=$ todos. En el tercer y último apartado, se incluía una escala que medía las representaciones sociales sobre el uso de drogas. Este instrumento estaba compuesto por seis ítems representativos 
de distintas creencias acerca del consumo social de drogas, seleccionados por expertos de entre un conjunto de creencias que más frecuentemente eran expresadas verbalmente por personas consumidoras de drogas, según la información recabada en distintos dispositivos sociales para el tratamiento de las drogodependencias ubicados en la provincia de Granada. Así, las creencias evaluadas fueron: (1) toda la gente consume drogas; (2) tomar alguna droga es normal; (3) si controlas no tiene porque pasarte nada; (4) deberían legalizar todas las drogas; (5) deberían legalizar algunas drogas y (6) tenemos que aprender a convivir con las drogas. La escala empleada para evaluar el grado con el que se identificaban los participantes con estas creencias fue de tipo Likert con cinco opciones de respuesta, donde 1: significaba nada, 2: poco, 3: regular, 4: bastante y 5: totalmente. El análisis de la fiabilidad del cuestionario se realizó mediante el coeficiente alpha de Cronbach, siendo la consistencia interna de 0.78.

\section{Procedimiento}

Los participantes cumplimentaron los cuestionarios de forma grupal y voluntaria en el aula, garantizando el anonimato. Se dieron las instrucciones para contestar las pruebas y se permaneció en el aula durante la aplicación.

\section{Diseño y análisis estadístico}

Se utilizó un diseño ex post facto. Se realizaron distintos contrastes sobre diferencias de dos me- dias, utilizando para tal efecto el estadístico $t$ de Student. También, se realizaron diferentes análisis de regresión simple y múltiple con el fin de conocer la capacidad predictiva de algunas de las variables sobre ciertos criterios. Así mismo, se utilizó análisis de correlaciones entre variables perceptivas de consumo y las creencias acerca de la amplitud de la representatividad social. El análisis se realizó con el programa SPSS .15.0.1.

\section{Resultados}

Las representaciones sociales que se han estudiado están presentes en la muestra evaluada. Examinando las creencias, consideradas como indicadores de la amplitud de las represtaciones sociales sobre el consumo de drogas, las dos primeras según la importancia concedida por los participantes son: "Toda la gente consume drogas" $(\mathrm{M}=2.91, \mathrm{DE}=$ 1.27) y "Deberían legalizar alguna droga" $(\mathrm{M}=$ $2.89, \mathrm{DE}=1.57$ ); creencias estas que consideramos como altamente favorecedoras de actitudes tendentes al consumo de drogas. Las otras cuatro creencias obtuvieron los siguientes valores: "Tomar alguna droga es normal" $(\mathrm{M}=2.57, \mathrm{DE}=1.43)$, "Si controlas no tiene porque pasarte nada" ( $M=2.48$, $D E=1.47)$, "Tenemos que aprender a convivir con las drogas" ( $\mathrm{M}=2.36, \mathrm{DE}=1.45)$ y "Deberían legalizar todas las drogas" $(M=2.01, D E=1.34)$. Teniendo en cuenta la totalidad de las respuestas, se observó que la mayoría de los encuestados se identificaban en alguna medida con estas creencias, excepto en el caso de la que hacía referencia a

TABLA 1

Diferencias entre fumadores y no fumadores en las distintas creencias sobre el consumo de drogas

\begin{tabular}{lccccc}
\hline & $\begin{array}{c}\text { Medias en } \\
\text { fumadores }\end{array}$ & $\begin{array}{c}\text { Medias en no } \\
\text { fumadores }\end{array}$ & $\begin{array}{c}t \text { de } \\
\text { Student }\end{array}$ & $g l$ & $p$ \\
\hline Toda la gente consume drogas & $2.21(1.24)$ & $3.2(1.26)$ & 3.224 & 402 & 0.001 \\
Tomar alguna droga es normal & $3.76(1.46)$ & $2.29(1.2)$ & 5.677 & 398 & 0.001 \\
Si controlas no tiene porque pasarte nada & $3.23(1.55)$ & $1.84(1.32)$ & 2.892 & 401 & 0.004 \\
Deberían legalizar todas las drogas & $2.68(1.42)$ & $1.9(1.22)$ & 2.82 & 405 & 0.005 \\
Deberían legalizar algunas drogas & $3.46(1.56)$ & $2.22(1.45)$ & 4.515 & 407 & 0.001 \\
Tenemos que aprender a convivir con las drogas & $3.89(1.49)$ & $2.35(1.27)$ & 4.862 & 392 & 0.001 \\
\hline
\end{tabular}

Nota. Fuma $(N=192)$ y no fuma $(N=225)$. Las desviaciones estándar están entre paréntesis.

Fuente: elaboración propia. 
"legalizar todas las drogas", ya que más de la mitad de los participantes no se identificaron con ella.

En general, parece que la identificación de los alumnos de la muestra con las distintas creencias no depende de la variable género, ya que no se encontraron diferencias estadísticamente significativas entre hombres y mujeres excepto en el caso de la creencia "Deberían legalizar algunas drogas" $\left(t_{412}=3.82, p=0.001\right)$. En esta creencia, los hombres $(M=3.52, D E=1.32)$ puntúan más alto que las mujeres $(\mathrm{M}=1.92, \mathrm{DE}=0.972)$. No se encontraron diferencias estadísticamente significativas en ninguna de las creencias en función de la variable edad.

Al comparar los grupos de fumadores y no fumadores en cada una de las creencias, se encontraron diferencias estadísticamente significativas. El mayor valor de las medias correspondió al grupo de personas que sí consumían tabaco a excepción de la creencia "Toda la gente consume drogas", donde el grupo de no fumadores presentaban una media superior. Si bien no era esperable este resultado, no obstante podría indicar la aceptación generalizada de la presencia de las drogas entre los jóvenes (Tabla 1).

Los participantes difieren en el grado de aceptación de las creencias sobre el uso social de las drogas dependiendo de si suelen emborracharse, al menos una vez al mes, o no se emborrachan nunca. Por lo general, los alumnos que se emborrachan puntuaron más alto en todas las creencias evaluadas, existiendo entre éstos y los que no lo hacían diferencias estadísticamente significativas exceptuando en la creencia "Deberían legalizar todas las drogas" (Tabla 2).

Se encontraron también diferencias estadísticamente significativas en todas las creencias al comparar los grupos que consumían al menos una vez al mes y no consumían cannabis, siendo en todas mayor el valor de la media de los ítems en el grupo de alumnos que consumían esta droga (Tabla 3). Esto podría demostrar que las creencias evaluadas son altamente significativas para el grupo de participantes que consumen cannabis, por lo que tales creencias podrían considerarse como representaciones de la imagen colectiva que este grupo tiene sobre el consumo social de ciertas drogas.

Cuando se comparó las creencias estudiadas entre los alumnos que consumían drogas ilegales y aquellos que no lo hacían, se observó que el valor de aquellos era mayor en los participantes que las consumían. Esto es, los que consumían drogas ilegales se identificaron significativamente más con estas creencias acerca del uso social de las drogas (Tabla 4).

También se analizó si tener amigos consumidores de drogas predecía el grado con el que los alumnos se identificaban con las creencias sobre el uso social de aquellas. Para ello, se realizó un análisis de regresión simple en el que la variable predictora era "Tener amigos que consumen drogas" y la variable criterio "Creencias sobre el uso social de

TABLA 2

Diferencias entre los participantes que se emborrachan y los que no lo hacen en las distintas creencias sobre el consumo de drogas

\begin{tabular}{lccccc}
\hline & $\begin{array}{c}\text { Medias síse } \\
\text { emborracha }\end{array}$ & $\begin{array}{c}\text { Medias no se } \\
\text { emborracha }\end{array}$ & $\begin{array}{c}t \text { de } \\
\text { Student }\end{array}$ & $g l$ & $p$ \\
\hline Toda la gente consume drogas & $3.15(1.21)$ & $2.63(1.29)$ & 3.023 & 411 & 0.003 \\
Tomar alguna droga es normal & $2.93(1.38)$ & $2.17(1.39)$ & 3.966 & 408 & 0.001 \\
Si controlas no tiene porque pasarte nada & $2.77(1.43)$ & $1.45(1.45)$ & 3.029 & 403 & 0.003 \\
Deberían legalizar todas las drogas & $2.13(1.34)$ & $1.87(1.33)$ & 1.39 & 409 & 0.166 \\
Deberían legalizar algunas drogas & $3.3(1.55)$ & $2.44(1.48)$ & 4.095 & 410 & 0.001 \\
Tenemos que aprender a convivir con las drogas & $2.61(1.49)$ & $2.05(1.36)$ & 2.844 & 407 & 0.005 \\
\hline
\end{tabular}

Nota. Se emborracha, al menos, una vez al mes $(N=201)$ y no se emborracha nunca $(N=216)$. Las desviaciones estándar están entre paréntesis.

Fuente: elaboración propia. 
TABLA 3

Diferencias entre consumidores de cannabis y no consumidores en las distintas creencias sobre el consumo de drogas

\begin{tabular}{lccccc}
\hline & $\begin{array}{c}\text { Medias sí } \\
\text { consume }\end{array}$ & $\begin{array}{c}\text { Medias no } \\
\text { consume }\end{array}$ & $\begin{array}{c}\mathrm{t} \text { de } \\
\text { Student }\end{array}$ & $g l$ & $p$ \\
\hline Toda la gente consume drogas & $3.46(1.02)$ & $2.54(1.29)$ & 5.743 & 411 & 0.001 \\
Tomar alguna droga es normal & $3.48(1.21)$ & $1.95(1.22)$ & 8.93 & 415 & 0.001 \\
Si controlas no tiene porque pasarte nada & $3.11(1.46)$ & $2.08(1.33)$ & 5.264 & 403 & 0.001 \\
Deberían legalizar todas las drogas & $2.48(1.4)$ & $1.7(1.21)$ & 4.204 & 405 & 0.001 \\
Deberían legalizar algunas drogas & $3.9(1.26)$ & $2.21(1.38)$ & 8.996 & 409 & 0.001 \\
Tenemos que aprender a convivir con las drogas & $3.22(1.42)$ & $1.81(1.2)$ & 7.442 & 412 & 0.001 \\
\hline
\end{tabular}

Nota. Consume cannabis al menos una vez al mes $(N=166)$ y no consumen $(N=251)$. Las desviaciones estandar están entre paréntesis.

Fuente: elaboración propia.

TABLA 4

Diferencias entre los consumidores de alguna droga ilegal y los no consumidores en las distintas creencias sobre el consumo de drogas

\begin{tabular}{|c|c|c|c|c|c|}
\hline & $\begin{array}{l}\text { Medias sí } \\
\text { consume }\end{array}$ & $\begin{array}{l}\text { Medias no } \\
\text { consume }\end{array}$ & $\begin{array}{c}t \text { de } \\
\text { Student }\end{array}$ & $g l$ & $p$ \\
\hline Toda la gente consume drogas & $3.43(1.03)$ & $2.52(1.3)$ & 5.73 & 412 & 0.001 \\
\hline Tomar alguna droga es normal & $3.44(1.17)$ & $1.92(1.25)$ & 9.021 & 410 & 0.001 \\
\hline Si controlas no tiene porque pasarte nada & $3.12(1.48)$ & $2.01(1.28)$ & 5.736 & 385 & 0.001 \\
\hline Deberían legalizar todas las drogas & $2.41(1.4)$ & $1.71(1.22)$ & 3.796 & 390 & 0.001 \\
\hline Deberían legalizar algunas drogas & $3.81(1.33)$ & $2.2(1.38)$ & 8.508 & 411 & 0.001 \\
\hline Tenemos que aprender a convivir con las drogas & $3.17(1.43)$ & $1.76(1.16)$ & 7.624 & 409 & 0.001 \\
\hline
\end{tabular}

Nota. Consume algún tipo de droga ilegal $(N=205)$ y no consume ninguna droga ilegal $(N=212)$. Las desviaciones estandar están entre paréntesis.

Fuente: elaboración propia.

drogas", representada mediante la media aritmética de las seis creencias evaluadas, pues la consistencia interna valorada mediante el coeficiente alpha de Cronbach fue de 0.74. Así, el análisis de regresión mostró que tener amigos consumidores de drogas predijo positivamente el grado con el que los participantes se identificaron con estas creencias $(\beta=0.47, p=0.001)$. En otras palabras, cuantos más amigos consumidores tenía la persona más se identificaba con las creencias acerca del uso social de las drogas, creencias estas que, a su vez, como hemos podido apreciar, estaban directamente relacionadas con el consumo de cannabis.

Para conocer si tener estas creencias predecía el consumo de cannabis, se realizó un análisis de regresión simple en el que la variable predictora fue "la media de todas estas creencias" y la variable criterio "consumo de cannabis", medida con la siguiente escala de valores: $1=$ nunca, $2=$ una vez al mes, $3=$ fines de semana, $4=$ de dos a seis veces en semana y $5=$ a diario. Los resultados indicaron que dichas creencias eran un predictor relevante para el consumo de cannabis $(\beta=0.56$, $\mathrm{p}=0.001$ ). Esto es, cuanto más se identificaron los participantes con estas creencias, más probable era que consumieran cannabis.

Para conocer el peso que tuvo cada una de estas creencias en la predicción del consumo de cannabis, se realizó un análisis de regresión múltiple en el que las seis variables predictoras fueron cada una de las creencias evaluadas y la variable criterio el consumo de cannabis con los cinco niveles de respuesta 
TABLA 5

Regresión múltiple de cada una de las creencias acerca del consumo de drogas sobre la frecuencia de consumo de cannabis

\begin{tabular}{lccccc}
\hline & \multicolumn{2}{c}{$\begin{array}{c}\text { Coeficientes } \\
\text { estandarizados }\end{array}$} & & & \multicolumn{2}{c}{$\begin{array}{c}\text { Estadísticos de } \\
\text { colinealidad }\end{array}$} \\
\cline { 2 - 6 } & $\beta$ & $t$ & $p$ & Tolerancia & FIV \\
\hline Toda la gente consume drogas & 0.142 & 2.302 & 0.022 & 0.779 & 1.283 \\
Tomar alguna droga es normal & 0.162 & 2.258 & 0.025 & 0.578 & 1.729 \\
Si controlas no tiene porque pasarte nada & -0.044 & -0.706 & 0.481 & 0.754 & 1.326 \\
Deberían legalizar todas las drogas & 0.003 & 0.041 & 0.967 & 0.677 & 1.478 \\
Deberían legalizar algunas drogas & 0.354 & 4.262 & 0.001 & 0.432 & 2.315 \\
Tenemos que aprender a convivir con las drogas & 0.146 & 2.223 & 0.027 & 0.686 & 1.458 \\
\hline
\end{tabular}

Fuente: elaboración propia.

TABLA 6

Relación entre la percepción sobre la cantidad de personas que consumen drogas ilegales en la ciudad y la media aritmética de todas las creencias acerca del consumo de drogas

\begin{tabular}{lcc}
\hline \multicolumn{1}{c}{ Consumo de drogas ilegales } & \multicolumn{2}{c}{ Media de las creencias } \\
\cline { 2 - 3 } & Correlación de Pearson & $p$ \\
\hline Personas de tu ciudad que fuman porros & $0.203^{\mathrm{a}}$ & 0.003 \\
Personas de tu ciudad que consumen drogas de diseño & $0.204^{\mathrm{b}}$ & 0.003 \\
Personas de tu ciudad que consumen cocaína & $0.246^{\mathrm{c}}$ & 0.001 \\
Personas de tu ciudad que consumen heroína & $0.141^{\mathrm{d}}$ & 0.045 \\
\hline
\end{tabular}

Nota. ${ }^{a} \mathrm{~N}=401 ;{ }^{\mathrm{b}} \mathrm{N}=405 ;{ }^{\mathrm{c}} \mathrm{N}=403 ;{ }^{\mathrm{d}} \mathrm{N}=402$.

Fuente: elaboración propia.

anteriores (Tabla 5). Según los resultados de este análisis, algunas de las creencias tenían más peso específico en la predicción que otras. La que más peso predictivo mostró fue "Deberían legalizar algunas drogas", probablemente porque el cannabis es una droga que, por su amplia aceptación social, se considera casi legal. Esta diferencia en los pesos podría estar motivada por el grado de riesgo percibido asociado a cada una de las drogas, influido esto a su vez por la percepción que los participantes tienen sobre el consumo social de las mismas.

Así mismo, en el desarrollo sociogénico de las representaciones sociales se sabe que intervienen distintos procesos para la racionalización explicativa de lo que las personas ven en su entorno. Esto es, la percepción de lo que sucede en nuestro ambiente social en relación con el consumo de drogas legales e ilegales podría influir en la formación del imaginario colectivo sobre su consumo. Para estudiar esto se analizó la relación entre la percepción que tenían los participantes sobre el consumo de drogas en su instituto y su ciudad, y el grado con el que se identificaron éstos con las creencias que se han descrito. La correlación bivariada entre las medias de los ítems que miden las distintas creencias y las puntuaciones referentes a la percepción sobre la incidencia de consumo de drogas en el instituto de los participantes no fue significativa. Al parecer, estas creencias, al menos en el ámbito de este estudio, no se construyen prioritariamente a partir de la percepción de incidencia del consumo de drogas en el instituto. Por el contrario, la percepción sobre la cantidad de personas que consumen drogas ilegales en la ciudad, un referente aparentemente más lejano para los participantes, si tenía una relación significativa con las creencias acerca del consumo de drogas. Así, un referente claro para la construcción de las creencias y, por lo tanto, de las representaciones sociales sobre el uso social de las drogas en los participantes, podría ser la percepción 
que se tiene sobre el número de personas que consumen drogas en la ciudad en la que habitualmente conviven (Tabla 6).

Esto se corroboró, en parte, tras la realización de un análisis de regresión múltiple, en el que las variables predictoras fueron la percepción de incidencia de porros en el instituto y la ciudad y como variable criterio el valor de la media aritmética de todas las creencias. Si bien la incidencia de consumo en el instituto no mostró capacidad predictiva sobre la variable criterio, sin embargo, sí la tuvo la percepción de la incidencia de consumo en la ciudad $(\beta=0.208, p=0.007)$. Cuantas más personas se percibe que consumen drogas en la ciudad mayor grado de identificación se produce con las creencias estudiadas.

\section{Discusión}

Creencias como "Toda la gente consume", "Tomar alguna droga es normal", "Si controlas no tiene porque pasarte nada", "Deberían legalizar algunas drogas" y "Tenemos que aprender a convivir con las drogas", son creencias muy extendidas entre los participantes del estudio, lo que corrobora que el consumo de drogas representa una opción de comportamiento bastante normalizado. Esto puede confirmar, por un lado, que el uso de drogas pueda llegar a ser un valor social positivo dependiendo del caso (Comas, 1994) y, por otro, dejar a la vista algunas características de las representaciones sociales, como lo son su homogeneidad y amplia presencia en los colectivos sociales (Wagner \& Alejabarrieta, 1995). Estas representaciones sociales, en las que no se observa, en general, diferencias a través de los niveles de las variables género y edad, y en cuya construcción parece tener una gran importancia la percepción de incidencia del consumo de drogas en el entorno macrosocial, están relacionadas con la conducta de consumo de drogas ilegales. El hecho de que tenga más relevancia la percepción de incidencia en la ciudad que en el instituto podría explicarse porque quizá los alumnos establecen lazos afectivos con personas fuera del instituto, no llegando a tener una vinculación emocional sufi- cientemente significativa con los compañeros del centro escolar.

Parece que emborracharse podría estar asociado con un riesgo mayor de consumo de cannabis (Vargas \& Trujillo, 2006), lo que estaría relacionado con tener creencias claras sobre la aceptación social de las drogas (Chabrol et al., 2006; Morales, Ariza, Nebot, Pérez \& Sánchez, 2008).

Por lo general, las personas que consumen drogas se identifican más que los no consumidores con las creencias estudiadas acerca del uso social de las mismas, lo que podría hacer pensar en la implicación de éstas en la emisión de conductas de consumo (Wagner \& Elejabarrieta, 1995). Además, tener amigos consumidores tiene un peso importante para comprender en toda su dimensión el fenómeno del uso de drogas entre los jóvenes (Chabrol et al., 2006; Ramos et al., 2011; Vargas \& Trujillo, 2012).

Así mismo, aunque parece que las actitudes no siempre predicen las conductas, en este estudio se ha podido observar, especialmente en lo que respecta al consumo de cannabis, coincidiendo con McCuller et al. (2001), que ciertas creencias favorecen actitudes proclives y anteriores al consumo. Por lo tanto, se podría pensar que las creencias y, así, las representaciones sociales tienen implicaciones directas sobre la conducta, ya que son moduladoras de actitudes favorables al uso de drogas. La cognición social tiene consecuencias directas en la conducta de consumo de las personas, ya que, según los resultados obtenidos en este estudio, la acumulación de ciertas creencias sobre la droga juega un papel predictivo respecto al consumo de la misma (Chabrol et al., 2006; Páez, Basabe, Igartua, Iraurgi \& Baldosada, 1992a).

No obstante todo lo anterior, las representaciones sociales acerca del uso de las drogas no se están teniendo en cuenta en los programas preventivos actuales a pesar de no haberse obtenido los resultados de eficacia esperados con su aplicación (Calafat et al., 1998). Una acción preventiva podría consistir, por ejemplo, en informar a los jóvenes que una gran parte de personas con edades similares desaprueba el uso de cannabis (Chabrol et al., 2006), o incrementar en los jóvenes la percepción del riesgo que 
entraña el consumo de drogas (Llorens, Barrio, Sánchez, Suelves \& ESTUDES Working Group, 2011).

El hecho de que las representaciones sociales tengan capacidad para predecir el consumo de cannabis convierte esta cuestión en un elemento clave para la puesta en marcha de estrategias de intervención preventiva, toda vez que las representaciones sociales, esto es, el imaginario colectivo de un determinado grupo de personas en torno al uso de drogas se convierte a todas luces en un buen predictor de su consumo.

Con respecto a los programas preventivos, es importante mencionar la dificultad asociada a las intervenciones que tienen como objetivo inducir modificaciones en el modo de pensar de un grupo de personas, sobre todo si estas sufren, además, trastornos de la personalidad (Martínez-González, Graña \& Trujillo, 2009, 2010). En el ámbito de las drogodependencias, sabemos que cuando las personas están experimentando con alguna droga, es altamente complicado hacerles llegar algún mensaje convincente y con capacidad de modificar las creencias sobre el tipo de riesgo que realmente están corriendo. Esto se podría deber a que las representaciones sociales tienen una verdad fiduciaria, generada por la confianza que se deposita en la información y los juicios, cuando aquella y éstos son compartidos con otras personas (Moscovici, 1988).

Se debe tener en cuenta que el núcleo de las representaciones sociales alberga la imagen que se tiene de una droga, lo que en definitiva queda asociado, entre otras cosas, al riesgo relacionado con su uso. Si se cree que tomar una droga no lleva consigo peligro alguno, se estará más cerca de hacer consumos experimentales que cuando la imagen se caracterice por la percepción de un elevado peligro asociado a su uso y que deberá ser objeto de evitación. Teniendo esto en cuenta, en toda intervención para la modificación de las representaciones sociales, en este caso sobre las consecuencias del consumo de las drogas, se tendrá que considerar el contenido de las creencias que las conforman. En ocasiones se persigue la modificación de las representaciones sociales sin éxito, como consecuencia de desconocer realmente el elemento más importante de las mismas, esto es, el núcleo. Pues bien, un modo de conocer el núcleo es indagando cómo piensan realmente los jóvenes y cuáles son las creencias que tienen.

Un aspecto a tener en cuenta en el presente estudio es que la muestra está conformada por alumnos considerados de alto riesgo para el consumo de drogas, lo que dificulta la posibilidad de generalizar los resultados a otros grupos con perfiles de consumo no considerados de alto riesgo. Además, al tratarse de un estudio transversal no es posible valorar la capacidad predictiva que pudiera tener el cambio de las creencias en el consumo de drogas a través de distintos momentos temporales. Por ello, sería aconsejable realizar nuevas investigaciones con muestras de alumnos que no sean de alto riesgo de consumo y se apliquen diseños longitudinales.

A modo de conclusión, se puede decir que este estudio ha permitido identificar ciertas creencias acerca del uso social de las drogas como indicadoras del contenido de las representaciones sociales sobre las mismas en adolescentes y adultos jóvenes. Los datos indican que las creencias estudiadas están muy extendidas entre los participantes, por lo que pueden constituirse en representaciones sociales sobre el consumo de las distintas drogas. Desde estas representaciones sociales se predice muy claramente el consumo de cannabis. Además, facilitan la homogeneidad entre las personas que las comparten, predicen el consumo de distintas drogas, $y$ favorecen que éste se perciba como algo totalmente aceptado entre los jóvenes.

\section{Referencias}

Basabe, N. \& Páez, D. (1992). Los jóvenes y el consumo de alcohol. Representaciones sociales. Bilbao: Fundamentos.

Becoña, E. (2002). Bases científicas de la prevención de las drogodependencias. Madrid: Delegación del Gobierno para el Plan Nacional sobre Drogas.

Calafat, A., Fernández, C., Juan, M., Anttila, A., Bellis, M., Bohrn, K., et al. (2003). Enjoying the nightlife in Europe. The role of moderation. Palma de Mallorca: Irefrea.

Calafat, A., Stocco, P., Mendes, F., Simon, J., Wijngaart, G., Sureda, M. P., et al. (1998). Characteristics and 
social representation of ecstasy in Europe. Palma de Mallorca: Irefrea.

Chabrol, H., Chauchard, E., Mabila, J., Montoulan, R., Adèle, A. \& Rousseau, A. (2006). Contributions of social influences and expectations of use to cannabis use in high school students. Addictive Behaviors, 31(11), 2116-2119.

Comas, D. (1994). Los jóvenes y el uso de drogas en la España de los años 90. Madrid: Ministerio de Asuntos Sociales, Instituto de la Juventud.

Day-Cameron, J. M., Muse, L., Hauenstein, J., Simmons, L. \& Correia, C. J. (2009). Alcohol use by undergraduate students on their 21st birthday: Predictors of actual consumption, anticipated consumption, and normative beliefs. Psychology of Addictive Behaviors, 23(4), 695-701.

Jessor, R. \& Jessor, S. L. (1977). Problem behavior and psychosocial developmental: A longitudinal study of youth. San Diego, CA: Academic Press.

Llorens, N., Barrio, G., Sánchez, A., Suelves, J. M. \& ESTUDES Working Group (2011). Effects of socialization and family factors on adolescent excessive drinking in Spain. Prevention Science, 12(2), 150-161.

Martínez-González, J. M., Graña, J. L. \& Trujillo, H. M. (2009). Influencia de los trastornos de la personalidad y patrones de consumo en la eficacia de un programa de prevención de recaídas para el tratamiento de alcoholismo. Adicciones, 21(2), 105-112.

Martínez-González, J. M., Graña, J. L. \& Trujillo, H. M. (2010). La calidad de vida en pacientes con trastorno por dependencia al alcohol con trastornos de la personalidad. Relación con el ajuste psicológico y "craving". Psicothema, 22(4), 562-567.

Martínez-González, J. M., Trujillo, H. M. \& RoblesLozano, L. (2007). Factores de riesgo, protección y representaciones sociales sobre el consumo de drogas: Implicaciones para la prevención. Sevilla: Consejería para la Igualdad y el Bienestar Social.

Martins, S. S., Storr, C. L., Alexandre, P. K. \& Chilcoat, H. D. (2008). Do adolescent ecstasy users have different attitudes towards drugs when compared to marijuana users? Drug and Alcohol Dependence, 94(1-3), 63-72.
McCuller, W. J., Sussman, S., Dent, C. W. \& Teran, L. (2001). Concurrent prediction of drug use among high-risk youth. Addictive Behaviors, 26(1), 137-142.

Megías, E. (1999, noviembre). Las representaciones sociales sobre drogas en España. Ponencia presentada en el VI Encuentro Nacional sobre Drogodependencias y su Enfoque Comunitario, Chiclana de la Frontera, España.

Megías, E., Comas, D., Elzo, J., Navarro, J. \& Romaní, O. (2001). La percepción social de los problemas de drogas en España. Madrid: Fundación de Ayuda Contra la Drogadicción.

Moral, M. V., Sirvent, C., Ovejero, A. \& Rodríguez, F. J. (2004). Comparación de la eficacia preventiva de programas de intervención psicosocial sobre las actitudes hacia el consumo juvenil de sustancias psicoactivas. Trastornos Adictivos, 6(4), 248-261.

Morales, E., Ariza, C., Nebot, M., Pérez, A. \& Sánchez, F. (2008). Consumo de cannabis en los estudiantes de secundaria de Barcelona: inicio en el consumo, efectos experimentados y expectativas. Gaceta Sanitaria, 22(4), 321-329.

Moscovici, S. (1986). Psicología social II. Pensamiento y vida social. Psicología social y problemas sociales. Barcelona: Paidós.

Moscovici, S. (1988). Notes towards a description of social representation. European Journal of Social Psychology, 18(3), 211-250.

Myers, D. G. (2000). Psicología social. Bogotá: McGrawHill.

Observatorio Europeo de las Drogas y las Toxicomanías. (2003). Informe anual sobre el problema de la drogodependencia en la Unión Europea. Luxembugo: Oficina de Publicaciones Oficiales de las Comunidades Europeas.

Páez, D., Basabe, N., Igartua, J., Iraurgi, J. \& Valdoseda, M. (1992a). Consumo de alcohol en jóvenes bilbaínos: motivos, representaciones sociales del alcohol y alternativas a su consumo. Bilbao: Universidad del País Vasco.

Páez, D., Basabe, N., Igartua, J., Iraurgi, J. \& Valdoseda, M. (1992b). Las representaciones sociales del alcohol. Revista de Psicología Social Aplicada, 2(2-3), 33-54. 
Pisano, S. \& Rooney, J. F. (1988). Children’s changing attitudes regarding alcohol: A cross-sectional study. Journal of Drug Education, 18(1), 1-11.

Pons, J., Berjano, E. \& García, F. (1999). Variables psicosociales que discriminan el consumo abusivo de alcohol en la adolescencia. Adicciones, 8(2), 177-191.

Ramos, P., Moreno, C., Rivera, F. \& López, A. (2011). The classification of Spanish adolescents based on substance consumption patterns and the analysis of the relationships within their social developmental contexts. The Spanish Journal of Psychology, 14(2), 734-745.

Szmigin, I., Bengry-Howell, A., Griffin, C., Hackley, C. \& Mistral, M. (2011). Social marketing, individual responsibility and the "culture of intoxication". European Journal of Marketing, 45(5), 759-779.

Trujillo, H. M., Oviedo-Joekes, E. \& Vargas, C. (2005). Anticipatory conditioned responses to subjective and physiological effects of heroin in addicted persons. International Journal of Clinical and Health Psychology, 5(3), 423-443.
Trujillo, H. M., Oviedo-Joekes, E. \& Vargas, C. (2006). Compensatory and mimetic conditioned responses to effects of heroin in addicted persons. Psicothema, 18(1), 59-65.

Vargas, C. \& Trujillo, H. M. (2006). Secuencia, asociación y riesgo de consumo de drogas legales e ilegales en alumnado universitario. Psicología Conductual, 14(1), 41-62.

Vargas, C. \& Trujillo, H. M. (2012). Cannabis consumption by female psychology students: The influence of perceived stress, coping and consumption of drugs in their social environment. Universitas Psychologica, 11(1), 119-130.

Wagner, W. \& Elejabarrieta, F. (1995). Representaciones sociales. En J. F. Morales (Coord.), Psicología social (pp. 816-841). Madrid: McGraw-Hill.

Wu, P., Liu, X. \& Fan, B. (2010). Factors associated with initiation of ecstasy use among US adolescents: Findings from a national survey. Drug and Alcohol Dependence, 106(2-3), 193-198. 
